\title{
TRIF is a regulator of TLR2-induced foam cell formation
}

\author{
BIN HUANG ${ }^{*}$, DAE-WEON PARK* ${ }^{*}$ and SUK-HWAN BAEK \\ Department of Biochemistry and Molecular Biology, College of Medicine, \\ Yeungnam University, Daegu 705-703, Republic of Korea
}

Received June 30, 2015; Accepted April 20, 2016

DOI: $10.3892 / \mathrm{mmr} .2016 .5647$

\begin{abstract}
The activation of toll-like receptor 2 (TLR2) stimulates foam cell formation, which is a key early event in the process of atherosclerosis. In the present study, the role of toll/interleukin-1 receptor-domain-containing adaptor-inducing interferon- $\beta$ (TRIF) in TLR2-mediated foam cell formation was investigated, and the importance of monocyte chemoattractant protein-1 (MCP-1), tissue factor (TF) and lectin-like oxidized low-density lipoprotein receptor-1 (Lox-1) were examined. Treatment of Raw 264.7 cells with the TLR2 agonist. $\mathrm{Pam}_{3} \mathrm{CSK}_{4}$, increased the gene expression of TRIF in a time-dependent manner (RT-PCR). The induced gene expression of TRIF stimulated by TLR2 was not observed in TLR2-knockout mice-derived bone marrow-derived macrophages (BMDMs). $\mathrm{Pam}_{3} \mathrm{CSK}_{4}$ increased the mRNA expression of TRIF in the wild-type BMDMs, but not in the TLR2-knockout BMDMs. Knockdown of the expression of TRIF using small interfering RNA decreased $\mathrm{Pam}_{3} \mathrm{CSK}_{4}$-induced foam cell formation (combination of oil-red $\mathrm{O}$ and hematoxylin staining), suggesting a role of TRIF. Stimulation of TLR2 increased the expression levels of various genes, which are known to control atherosclerosis, including MCP-1, TF and Lox-1. The knockdown of TRIF also attenuated the $\mathrm{Pam}_{3} \mathrm{CSK}_{4}$-induced expression of these genes. In
\end{abstract}

Correspondence to: Professor Suk-Hwan Baek, Department of Biochemistry and Molecular Biology, 210 Main Building, College of Medicine, Yeungnam University, 170 Hyeonchung-ro, Nam-gu, Daegu 705-703, Republic of Korea

E-mail: sbaek@med.yu.ac.kr

*Contributed equally

Abbreviations: TLR, toll-like receptor; MyD88, myeloid differentiation factor 88 ; NF- $\kappa \mathrm{B}$, nuclear factor- $\kappa \mathrm{B}$; IRF, interferon regulatory factor; $\mathrm{MCP}-1$, monocyte chemoattractant protein-1; TRAF, tumor necrosis factor receptor-associated factor; BMDM, bone marrow-derived macrophage; siRNA, small interfering RNA; TF, tissue factor; Lox-1, lectin-like oxidized low-density lipoprotein receptor-1; LDL, low density lipoprotein

Key words: toll-like receptor 2, toll/interleukin-1 receptor-domain containing adaptor-inducing interferon- $\beta$, myeloid differentiation factor 88 , foam cell, monocyte chemoattractant protein-1 addition, a reduction in TRIF affected the $\mathrm{Pam}_{3} \mathrm{CSK}_{4}$-induced protein expression of MCP-1 (EIA). Taken together, the results of the present study suggested that TRIF regulated foam cell formation via regulation of the expression levels of MCP-1, TF and Lox-1.

\section{Introduction}

Atherosclerosis is a chronic inflammatory disorder in which metabolic and immune components interact to initiate and develop arterial lesions (1). Macrophages are the major immune cells in arterial lesions, which release various inflammatory cytokines and chemokines, are involved in foam cell formation and express pattern-recognition receptors, including, toll-like receptors (TLRs, which mediate innate and adaptive immune responses (2). There is substantial evidence that the stimulation of TLRs initiates and accelerates atherosclerosis (3). Of the TLRs involved in atherosclerosis, TLR2 and TLR4 have received the most investigation, and 11 and 13 of these receptors have been identified in humans and mice, respectively (4). However, the pathways that link TLRs with cytoplasmic adaptors, including toll/interleukin-1 receptor-domain-containing adaptor-inducing interferon- $\beta$ (TRIF) in the process of atherosclerosis remain to be fully elucidated.

TLR signaling is controlled by four types of cytoplasmic adaptors, myeloid differentiation factor 88 (MyD88), TRIF, MyD88 adaptor-like (MAL) and TRIF-related adaptor molecule (TRAM). During TLR4 signaling, MyD88 and MAL are recruit for the first signaling pathway, leading to early nuclear factor $-\kappa \mathrm{B}(\mathrm{NF}-\kappa \mathrm{B})$ activation and the induction of cytokine genes (5). By contrast, TRIF and TRAM are recruited for the second signaling pathway, which activates interferon regulatory factor (IRF)3, late NF- $\kappa \mathrm{B}$ activation and induction of the interferon gene (6). Although TLR2 signaling also uses MyD88 and Mal in a similar manner to TLR4, the availability of TRIF remains to be elucidated. During TLR3 and TLR4 signaling, TRIF initiates a signaling pathway through TNF receptor associated factor (TRAF)3, TANK-binding kinase 1 and inhibitor of NF- $\kappa \mathrm{B}-\mathrm{kinase}$ complex, which mediates the direct phosphorylation of IRF3 and IRF7 (7).

Several studies have focused on the role of MyD88 in atherosclerosis. Inactivation of MyD88 leads to a reduction in atherosclerosis mediated by reduced macrophage recruitment to the artery wall, which is associated with reduced chemokine levels (8). Additionally, Michelsen et al showed that apolipoprotein $\mathrm{E}^{-/-} \mathrm{MyD} 88^{-/-}$mice exhibit reduced aortic 
atherosclerosis and reduced macrophage accumulation (9). However, the role of TRIF in atherosclerosis remains to be fully elucidated, although a previous study by Lundberg et al demonstrated that the deletion of TRIF from myeloid cells was sufficient to attenuate vessel inflammation and protect against atherosclerosis (1). However, the role of TRIF in foam cell formation mediated by TLR2 stimulation remains to be fully elucidated.

Considering the significant importance of foam cell formation in TLR2 signaling, we aimed to determine whether TRIF is induced by TLR2 stimulation and whether TRIF is involved in foam cell formation in macrophages and if so, to determine the molecular mechanisms involved. Taken together, these results suggested the importance of TRIF in TLR2 mediated foam cell formation via inflammatory mediators, including MCP-1.

\section{Materials and methods}

Materials. $\mathrm{Pam}_{3} \mathrm{CSK}_{4}$ and the mouse TLR1-9 agonist kit was purchased from InvivoGen (San Diego, CA, USA). TRIzol and small interfering (si)RNA (TRIF) were purchased from Invitrogen; Thermo Fisher Scientific, Inc., Waltham, MA, USA).

Animals. A total of 20 male 6-week-old C57BL6 wild-type (WT) mice (average weight) were purchased from Central Lab Animal (South Korea). TLR2 deficient (average weight, C57BL6 background, 6 weeks, male) mice were kindly provided by Dr SJ Lee (Seoul National University, South Korea). For the experiments, a total of 20 mice were used. The mice were housed in standard conditions (temperature at $25 \pm 2^{\circ} \mathrm{C}$, relative humidity $(55 \pm 5 \%), 12 / 12 \mathrm{~h}$ light-dark cycle, and free access to food and water). The mice were sacrificed by cervical dislocation. The study was conducted in accordance with the guidelines and protocols approved by the Institutional Animal Care and Use Committee of Yeungnam University College of Medicine (Daegu, South Korea, permit number: YUMC-AEC2011-007).

Cell culture. Raw 264.7 cell lines were purchased from the American Type Culture Collection (Manassas, VA, USA) and grown in Dulbecco's modified Eagle's medium (DMEM) supplemented with $10 \%$ fetal bovine serum, penicillin (100 U/ml) and streptomycin $(100 \mu \mathrm{g} / \mathrm{ml}$; GE Healthcare Life Sciences, Logan, UT, USA) at $37^{\circ} \mathrm{C}$ in a humidified atmosphere of $5 \% \mathrm{CO}_{2}$ and $95 \% \mathrm{O}_{2}$. After euthanasia, the mice were sprayed with $70 \%$ ethanol and the femurs were dissected using scissors, cutting through the tibia below the knee joints, and through the pelvic bone close to the hip joint. Muscles connected to the bone were removed using clean gauze, and the femurs were placed into a polypropylene tube containing sterile phosphate-buffered saline (PBS) on ice. In a tissue culture hood, the bones were placed in $70 \%$ ethanol for $1 \mathrm{~min}$, washed in sterile DMEM and then both epiphyses were removed using sterile scissors and forceps. The bones were flushed with a syringe filled with DMEM to extrude bone marrow into a $15 \mathrm{ml}$ sterile polypropylene tube. A $5 \mathrm{ml}$ plastic pipette was used to gently homogenize the bone marrow. Primary bone marrow-derived monocytes were differentiated into bone marrow-derived macrophages (BMDMs) by incubation in DMEM supplemented with 10\% L929 cell (ATCC, Manassas, VA, USA)-conditioned medium, as a source of macrophage colony-stimulating factor, for $5-7$ days at $37^{\circ} \mathrm{C}$ in a humidified atmosphere of $5 \% \mathrm{CO}_{2}$ and $95 \% \mathrm{O}_{2}$. The macrophages were cultured in $35 \mathrm{~mm}$ diameter plates (or 6 -well plates) $\left(5 \times 10^{5} / 1.5 \mathrm{ml}\right.$ medium $)$ and then treated with Pam3CSK4 (100 ng/ml) for 2-6 h.

Electroporation. The Raw 264.7 cells were cultured in $100 \mathrm{~mm}$ diameter dishes at $1 \times 10^{6}$ and grown overnight prior to electroporation using Amaxa Cel Line Nuclofector Kit V (VCA-1003, Lonza, Switzerland). Cell were harvested and a defined number of cells washed with PBS $\left(2 \times 10^{6}-4 \times 10^{6}\right.$ cells per electroporation). The cells were pelleted by centrifugation (100 x g for $2 \mathrm{~min}$ ) and resuspended in $100 \mu \mathrm{l}$ of the electroporation solution provided with the kit. A total of $8 \mu \mathrm{l}$ of the siRNA $(150 \mathrm{pM})$ was added to the cell suspension and gently mixed. The cell-siRNA mix was transferred into the Amaxa electroporation cuvette, placed into the Nuclofector and electroporated as described by the manufacturer's instruction. Subsequently, $500 \mu \mathrm{l}$ warm media was added to the cuvette, and the sample transfered to the plate and incubated at $37^{\circ} \mathrm{C}$.

Reverse transcription-polymerase chain reaction (RT-PCR) and RT-quantitative PCR (RT-qPCR). Total RNA was extracted from the cells using TRIzol reagent. First-strand cDNA was synthesized from $1 \mu \mathrm{g}$ total RNA by employing random primers, oligo-dT and reverse transcriptase (Promega Corporation, Madison, WI, USA). The thermal cycling condition were as follows: $95^{\circ} \mathrm{C}$ for $5 \mathrm{~min}, 95^{\circ} \mathrm{C}$ for $1 \mathrm{~min}, 63^{\circ} \mathrm{C}$ for $1 \mathrm{~min}$, and $72^{\circ} \mathrm{C}$ for $1 \mathrm{~min}$, for 26-33 cycles, using a Bio-Rad C1000 ${ }^{\mathrm{TM}}$ Thermal Cycler (Bio-Rad Laboratories, Inc., Hercules, CA, USA). A total of $10 \mu \mathrm{l}$ of the final amplification product were electrophoresed on a $2 \%$ agarose gel containing SYBR ${ }^{\circledR}$ Safe DNA gel stain (Thermo Fisher Scientific, Inc.). The expression levels of TLR2, TRIF, MCP-1, LOX-1 and TF were normalized against the $\beta$-actin control and visualized using the Fuji Intelligent Dark Box LAS-3000 Image Reader (FujiFilm, Tokyo, Japan). Densitometric analysis was carried out using LAS-3000 Fujifilm Image Reader and Multi Gauge 3.0 software. The following primers were used for the RT-qPCR: TRIF, forward 5'-GTATGGGCCCTCTGACTG AT-3' and reverse 5'-ATAGGTGTG GTCTTCCCTGC-3'; MCP-1, forward 5'-AGAGAGCCAGACGGGAGGAA-3' and reverse 5'-GTCACACTGGTCACTCCTAC-3'; TF, forward 5'-CACTCATCATTGTGGGAGCAGTG-3' and reverse 5'-CGCGACGGGGTGTTCTT-3'); Lox-1, orward 5'-AGG TCCTTGTCCACAAGACTGG-3' and reverse 5'-ACGCCC CTGGTCTTAAAGAATTG-3'); and $\beta$-actin, forward 5'-TCC TTCGTTGCCGGTCCACA-3' and reverse 5'-CGTCTCCGG ACTCCATCACA-3'.

siRNA. Stealth control siRNA and gene-specific siRNA against the following target gene were designed using Block-IT Stealth RNAi designer (Invitrogen; Thermo Fisher Scientific, Inc.): TRIF, 5'-GGACAUACGUUACACUCCACCAACA-3'.

Oil-red $O$ and hematoxylin staining for foam cell formation. For lipid uptake analysis, the macrophages were cultured in 
A

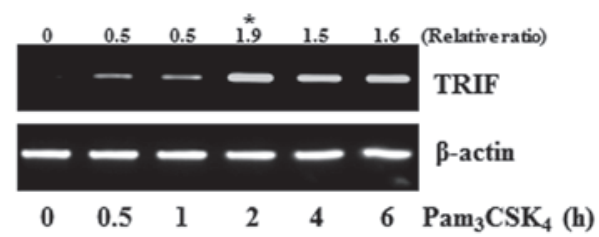

$\mathbf{B}$

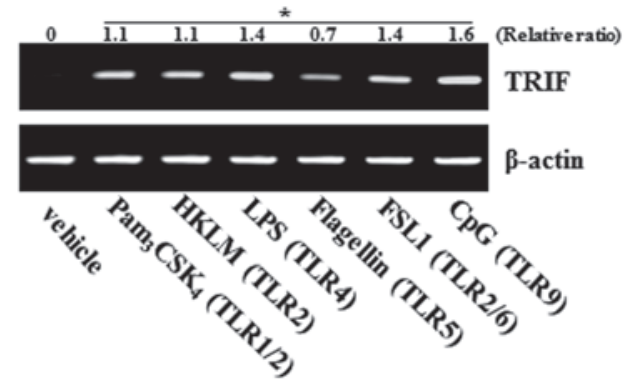

C

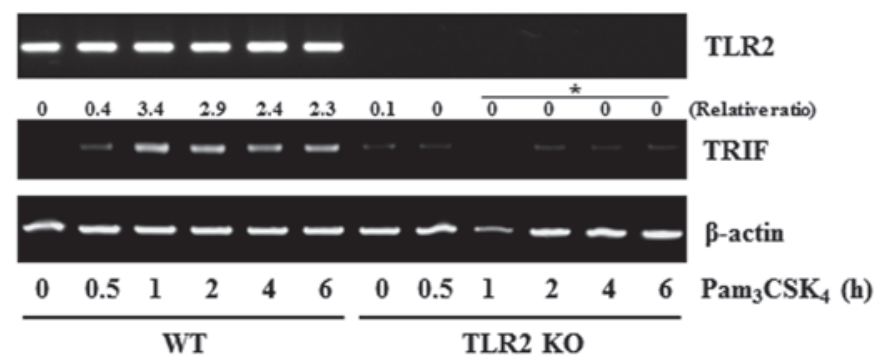

Figure 1. $\mathrm{Pam}_{3} \mathrm{CSK}_{4}$ induces the expression of TRIF via a TLR2-dependent pathway. (A) Raw 264.7 cells were treated with Pam ${ }_{3} \mathrm{CSK}$ (100 ng/ml) for the indicated times, following which the mRNA expression levels of TRIF were determined using RT-qPCR analysis. (B) Raw 264.7 cells were treated with various TLR agonists for $2 \mathrm{~h}$, following which the mRNA expression levels of TRIF were determined using RT-qPCR analysis. (C) Bone marrow-derived macrophages were isolated from the bone marrow of WT or TLR2 KO mice and treated with Pam ${ }_{3} \mathrm{CSK}_{4}(100 \mathrm{ng} / \mathrm{ml})$. The mRNA expression levels of TLR2 and TRIF were determined using RT-qPCR analysis. Representative results are shown from triplicate experiments with similar results. Values are presented as the mean, $\mathrm{P}<0.05$ vs. the control. TRIF, toll/interleukin-1 receptor-domain-containing adaptor-inducing interferon- $\beta$; TLR2, toll-like receptor 2 ; RT-PCR, reverse transcription-polymerase chain reaction; WT, wild-type; KO, knockout.

6-well plates $\left(5 \times 10^{5} /\right.$ well) and then treated with $\mathrm{Pam}_{3} \mathrm{CSK}_{4}$ $(100 \mathrm{ng} / \mathrm{ml})$ and low density lipoprotein LDL $(50 \mu \mathrm{g} / \mathrm{ml})$ for $24 \mathrm{~h}$ at $37^{\circ} \mathrm{C}$ in a humidified atmosphere of $5 \% \mathrm{CO}_{2}$ and $95 \% \mathrm{O}_{2}$. Prior to Oil-red $\mathrm{O}$ staining, the media were removed from the wells using a Pasteur pipette or by gently inverting the plate over a waste container, and then gently rinsing with phosphate-buffered saline. Subsequently, $10 \%$ formalin was added to each well for $1 \mathrm{~h}$ to fix the cells, following which each well was rinsed with distilled $\mathrm{H}_{2} \mathrm{O}$. The wells were then rinsed with $60 \%$ isopropanol for $5 \mathrm{~min}$, dried and stained with Oil-red O (Sigma-Aldrich, St. Louis, MO, USA) and hematoxylin. Intracellular lipid droplets were detected by light microscopy using a DIAPHOT 300 light microscope (Nikon Corporation, Tokyo, Japan). Images were captured with an AxioCam ICc I digital camera system (Carl Zeiss, Oberkochen, Germany).

Enzyme-linked immunosorbent assay (EIA). The protein levels of MCP-1 in the cell culture supernatant were measured by EIA using a specific sandwich enzyme immunoassay (mouse CCL2/JE/MCP-1 DuoSet ELISA kit; DY479, R\&D Systems, Inc., Minneapolis, MN, USA). Briefly, the cell culture supernatant was placed in a 96-well microtiter plate, which was coated with murine polyclonal antibody against mouse MCP-1. Following incubation at room temperature for $2 \mathrm{~h}$ and careful washes, HRP-conjugated polyclonal antibody against MCP-1 was added. Following incubation for $2 \mathrm{~h}$ at room temperature and repeated washes, color reagents were added. The optical density of each well was then measured at $450 \mathrm{~nm}$ using an EL800 universal microplate reader (Bio-Tek instruments, Inc., Winooski, VT, USA) using a $550 \mathrm{~nm}$ reference wavelength.

Statistical analysis. Results are expressed as the mean \pm standard deviation of a minimum of 3 independent assays. Statistical significance was calculated by analysis of variance using GraphPad Prism software, version 5.01 (GraphPad. Inc., La Jolla, CA, USA). Groups were compared with two-way analysis of variance with a Bonferroni post-test. $\mathrm{P}<0.05$ was considered to indicate a statistically significant difference.

\section{Results}

Gene expression of TRIF is induced by TLR activation. MyD88 and TRIF are well known adaptor proteins involved in TLR4 signaling, however, only MyD88 is involved in TLR2 signaling (10). The present study investigated whether the TLR2 agonist, $\mathrm{Pam}_{3} \mathrm{CSK}_{4}$, can induce the mRNA expression of TRIF in the Raw 264.7 macrophage cell line. The results revealed that $\mathrm{Pam}_{3} \mathrm{CSK}_{4}$ induced the mRNA expression of TRIF in a time-dependent manner (Fig. 1A). To demonstrate the general effect of TLRs on the expression of TRIF, other 
A

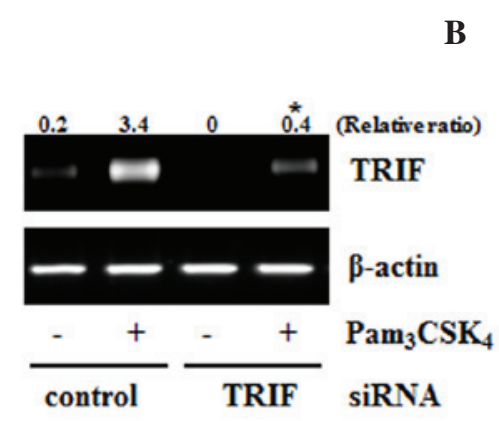

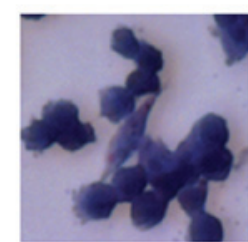

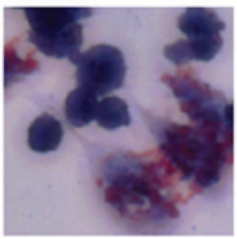

Control

siRNA
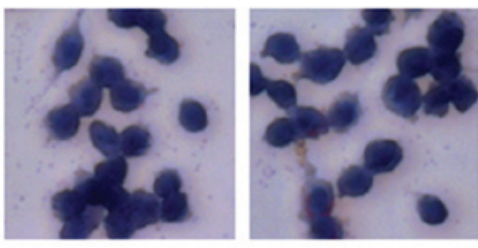

$+$
TRIF

SiRNA

\section{$\mathrm{Pam}_{3} \mathrm{CSK}_{4}$}

Figure 2. TRIF is an important adaptor protein in TLR2-mediated foam cell formation. (A) Raw 264.7 cells were transfected with either control siRNA or TRIF siRNA $(150 \mathrm{pM})$ for $24 \mathrm{~h}$, following which the cells were stimulated with vehicle or $\mathrm{Pam}_{3} \mathrm{CSK}_{4}$ for $2 \mathrm{~h}$. The mRNA expression levlels of TRIF were determined using reverse transcription-polymerase chain reaction analysis and normalized against the $\beta$-actin control. (B) Raw264.7 cells were transfected with either control siRNA or TRIF siRNA for $24 \mathrm{~h}$ (magnification, $\mathrm{x} 400$ ). The siRNA-transfected cells were incubated with $\mathrm{Pam}_{3} \mathrm{CSK}_{4}$ in the presence of low density lipoprotein for $24 \mathrm{~h}$ and stained with Oil-red O (red) and hematoxylin (blue). Representative results are shown from experiments repeated in triplicate with similar results. Values are presented as the mean, $\mathrm{P}<0.05$ vs. the control. TRIF, toll/interleukin-1 receptor-domain-containing adaptor-inducing interferon- $\beta$; TLR2, toll- like receptor 2 ; siRNA, small interfering RNA.

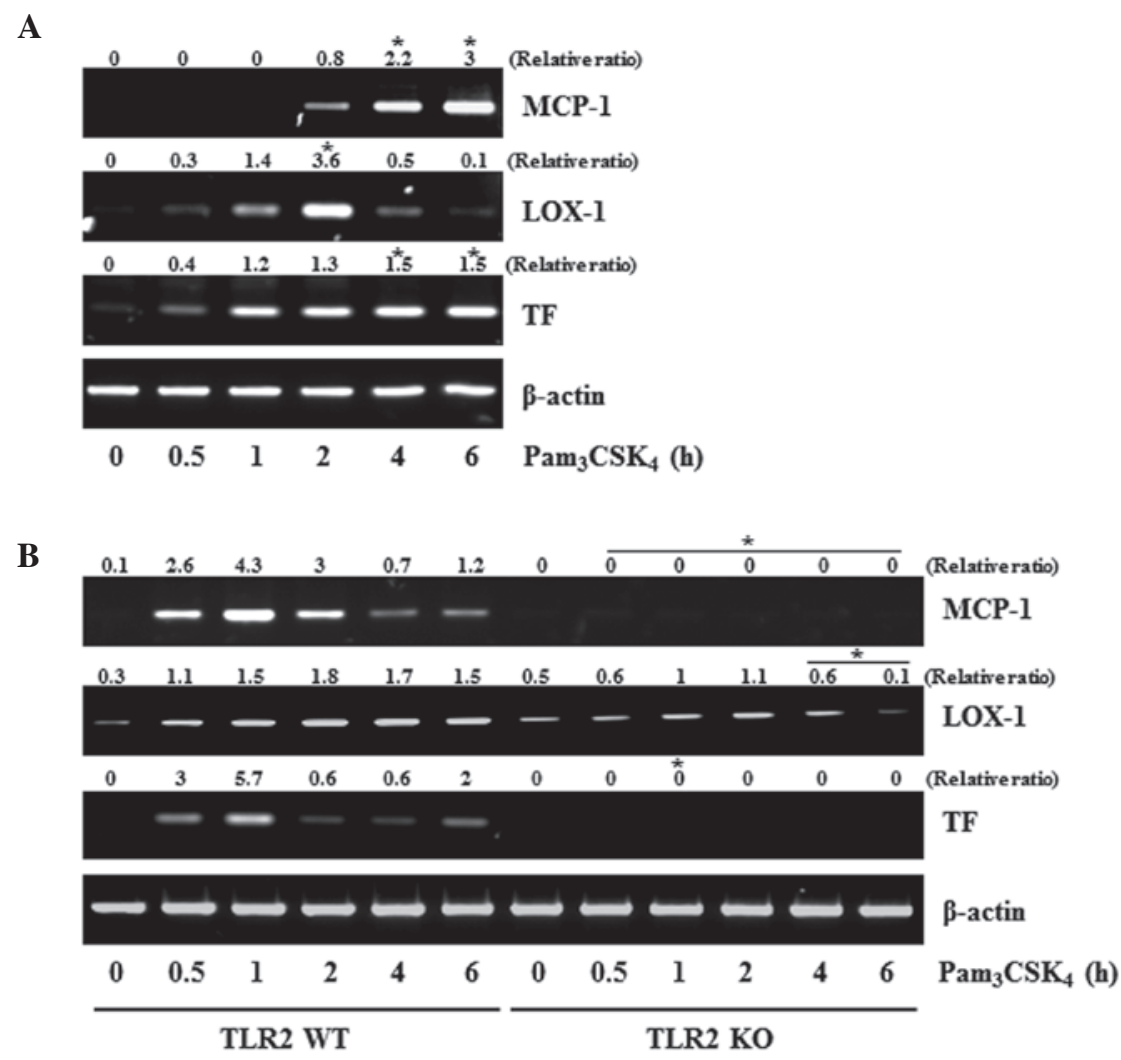

Figure 3. $\mathrm{Pam}_{3} \mathrm{CSK}_{4}$ induces inflammatory mediators via a TLR2-dependent pathway. (A) Raw 264.7 cells were treated with Pam ${ }_{3} \mathrm{CSK}_{4}(100 \mathrm{ng} / \mathrm{ml})$ for the indicated times. The mRNA expression levels of MCP-1, TF and Lox-1 were determined using RT-PCR analysis and normalized against the $\beta$-actin control. (B) BMDMs were isolated from the bone marrow of WT or TLR2 KO mice and treated with $\mathrm{Pam}_{3} \mathrm{CSK}_{4}(100 \mathrm{ng} / \mathrm{ml})$ for the indicated times. The expression gene expression levels of MCP-1, TF and Lox-1 genes were determined using RT-PCR analysis. The results shown are representative of experiments repeated in triplicate with similar results. Values are presented as the mean, $\mathrm{P}<0.05$ vs. the control. TRIF, toll/interleukin-1 receptor-domain-containing adaptor-inducing interferon- $\beta$; TLR2, toll-like receptor 2; MCP-1, monocyte chemoattractant protein-1; TF, tissue factor; Lox-1, lectin-like oxidized low-density lipoprotein receptor-1; RT-PCR, transcription-quantitative polymerase chain reaction; WT, wild-type; KO, knockout.

types of TLR agonist were assessed. All of the agonists examined (TLR1/2, TLR4, TLR5, TLR6/2 and TLR9) induced the gene expression of TRIF, with effects similar to those of the TLR2 agonist (Fig. 1B). These results suggested that the gene expression of TRIF by TLR agonists is a general characteristic shown in TLRs. Whether $\mathrm{Pam}_{3} \mathrm{CSK}_{4}$ affects the gene expression of TRIF was also determined in BMDMs. The gene expression of TRIF was induced by $\mathrm{Pam}_{3} \mathrm{CSK}_{4}$ in the BMDMs 
A

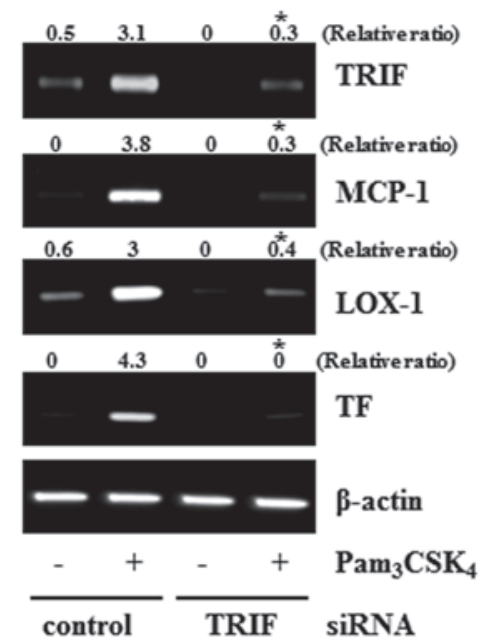

B

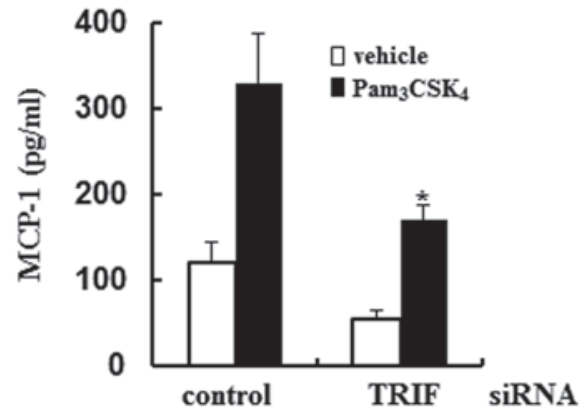

Figure 4. Production of inflammatory mediators occurs in a TRIF-dependent manner. Raw 264.7 cells were transfected with either control siRNA or TRIF siRNA (150 pM) for $24 \mathrm{~h}$. The cells were then stimulated with vehicle or $\mathrm{Pam}_{3} \mathrm{CSK}_{4}$ for 2 or $6 \mathrm{~h}$. (A) Gene expression levels of TRIF, and of MCP-1, TF and Lox-1 were determined using reverse transcription-polymerase chain reaction analysis and normalized against the $\beta$-actin control. (B) Protein levels of MCP-1 in the cell culture supernatant were measured using an enzyme-linked immunosorbent assay. ${ }^{*} \mathrm{P}<0.05$ vs. the control. The results shown are representative of experiments repeated in triplicate with similar results. Values are presented as the mean \pm standard deviation. TRIF, toll/interleukin-1 receptor-domain-containing adaptor-inducing interferon- $\beta$; MCP-1, monocyte chemoattractant protein-1; TF, tissue factor; Lox-1, lectin-like oxidized low-density lipoprotein receptor-1; siRNA, small interfering RNA.

from the WT mice, however, its expression was not affected in the BMDMs from the TLR2-knockout mice (TLR2 KO; Fig. 1C).

TRIF is involved in TLR2-induced foam cell formation. Previous studies have suggested that TLRs is involved in the pathology of atherosclerosis $(11,12)$. Among TLRs, TLR2 is also involved in the progression of atheroma. A previous study reported that TLR2 is a receptor for foam cell formation $(13,14)$; therefore, the present study attempted to determine the role of TRIF in TLR2-mediated foam cell formation using the siRNA technique. Compared with the control siRNA-transfected cells, the TRIF-siRNA transfected cells showed decreased mRNA expression of TRIF. The TLR2-mediated foam cell formation also decreased in the TRIF siRNA-transfected cells, compared with the control cells (Fig. 2A and B). These results suggested that TRIF was an important adaptor for TLR2-mediated foam cell formation.

Inflammatory mediators are upregulated by TLR 2 stimulation. TRIF is also likely to be important adaptor for TLR2-mediated foam cell formation. To identify genes associated with TRIF, the present study aimed to identify genes, which control foam cell formation. Chemokines are involved in the pathogenesis of atherosclerosis by promoting the directed migration of inflammatory cells. MCP-1 is a representative chemokine involved in foam cell formation (15). In the present study, treatment of the cells with $\mathrm{Pam}_{3} \mathrm{CSK}_{4}$ markedly increased the mRNA expression of MCP-1 in a time-dependent manner. TF is a major risk factor for atherosclerosis, and lectin-Lox-1 is also crucial in oxidized-LDL-mediated atherosclerosis $(14,16)$. Therefore, the present study measured changes in the expression levels of these two genes following TLR2 stimulation. The expression levels of the two genes increased significantly, in a time-dependent manner, although the reaction time was marginally different for the expression of MCP-1 (Fig. 3A). The present study also confirmed whether these genes were dependent on TLR2 using $\mathrm{KO}$ mice-derived BMDMs. Whereas the expression levels of the genes were induced by $\mathrm{Pam}_{3} \mathrm{CSK}_{4}$ in the BMDMs from the WT mice, their expression levels remained unchanged in the BMDMs from the TLR2-knockout mice (Fig. 3B).

Expression levels of MCP-1, TF and Lox-1 are TRIF dependent. The present study used siRNA to confirm the role of TRIF in the expression of MCP-1, TF and Lox-1. The TRIF siRNA technique was found to be successful in downregulation its level of gene expression. With the downregulation in the level of TRIF, the gene expression levels of MCP-1, TF and Lox-1 were also markedly attenuated on examination of the $\mathrm{Pam}_{3} \mathrm{CSK}_{4}$-induced responses (Fig. 4A). To confirm the changes in MCP-1, the present study assessed the protein levels of MCP-1 using an EIA. Production of the MCP-1 protein increased $\sim 3.5$-fold in the supernatants of the control siRNA-transfected cells following TLR2 stimulation. However, the protein expression of MCP-1 was reduced to almost basal levels in the TRIF siRNA-transfected cells (Fig. 4B). These results suggested that TRIF was an important adaptor protein, which controlled foam cell formation via the production of MCP-1.

\section{Discussion}

The present study initially found that $\mathrm{Pam}_{3} \mathrm{CSK}_{4}$ treatment increased the gene expression of TRIF in macrophages, suggesting that TRIF contributed to TLR 2 signaling. TLR 2 has previously been shown to be regulated via a MyD88-dependent signaling pathway only. However, the present study found that the TRIF adaptor protein also appeared to be a regulator of TLR2-mediated foam cell formation. In addition, as TRIF controls the expression of MCP-1, TF and Lox-1, which are 
representative regulators of foam cell formation, the present study hypothesized that TRIF was also an important adaptor protein in TLR2-mediated foam cell formation, together with MyD88.

TLR signaling is divided into an MyD88-dependent pathway and an MyD88-independent signaling pathway (17). The MyD88-dependent signaling pathway is used by all TLRs, with the exception of TLR3. Signaling via the MyD88-dependent pathways leads to the activation of mitogen-activated protein kinase and the inhibitor of NF- $\mathrm{\kappa B}$ (IאB) kinase complex, resulting in activation of activator protein (AP)-1 and NF- $\mathrm{KB}$, respectively $(18,19)$. By contrast, TLR3 is only present in the MyD88-independent pathway. TRIF is the predominant adaptor protein in the MyD88-independent pathway, and can associate with TRAF6 to activate AP-1 and $\mathrm{NF}-\mathrm{\kappa B}$, as in the MyD88-dependent pathway. TRIF can also interact with TRAF3 and phosphoinositide 3-kinase, resulting in the activation of interferon regulatory factor (IRF) 3 and IRF2, respectively $(20,21)$. TLR4 uses MyD88 and TRIF as adaptor proteins $(22,23)$.

Generally, TRIF is constantly expressed in macrophages to convey signals via the interaction with downstream proteins, inducing various functions through the activation of specific transcription factors. However, the results of the present study showed that TLR2 stimulation elevated the gene expression of TRIF itself. To date, few mechanisms have been shown to increase the gene expression levels of TRIF. In the present study, the gene expression levels of TRIF were upregulated by all the TLR agonists assessed, indicating that this may be a general observation in TLR signaling. Due to the TRIF antibody quality, the present study was only able to verify the increase in gene levels. $\mathrm{Pam}_{3} \mathrm{CSK}_{4}$ treatment increased the expression of TRIF, along with other inflammatory mediators, whereas the downregulation of TRIF by siRNA decreased foam cell formation and inflammatory mediators. Therefore, the changes in the levels of TRIF may be an essential factor stimulating foam cell formation. In addition, the downregulation of TRIF decreased the expression levels of MCP-1, TF and Lox-1, which regulate foam cell formation. These three genes are known to be important mediators involved in atherosclerosis through foam cell formation (14-16).

MCP-1, which is also referred to as CCL2, is a small cytokine, which belongs to the CC chemokine family. MCP-1 is one of the key chemokines involved in the regulation of migration and infiltration of monocytes/macrophages $(24,25)$. TF, which is the key initiator of the coagulation cascade, binds factor VIIa, resulting in activation of factor IX and factor X, ultimately leading to fibrin formation. TF is involved in the pathogenesis of atherosclerosis by promoting thrombus formation (26). Oxidized LDL is also crucial in the initiation and progression of atherosclerosis through a variety of mechanisms, including promoting foam cell generation and activating inflammatory processes (27). Lox-1, a type II membrane protein with a typical C-type lectin structure, has been identified as the predominant receptor for oxidized-LDL (28). Reported data have revealed that Lox-1 is important in atherosclerosis (16). On examination of these three factors, which are essential in atherosclerosis, their gene expression levels were reduced by TRIF knockdown, suggesting the presence of a direct association between TRIF and foam cell formation. Therefore, when the expression of TRIF is increased by TLR2 stimulation, inflammatory mediators, including MCP-1, produce and eventually promote the conversion of macrophages into foam cells. Therefore, the results of the present study showed that the reduction in expression levels of the above genes by TRIF knockdown was directly associated with foam cell formation.

The production of these three genes regulating foam cell formation is known to be regulated by the MyD88-dependent pathway in TLR signaling. However, the results of the present study showed that these three genes were regulated in a TLR2/TRIF-dependent manner. Associated reports have shown that the TLR4/TRIF pathway, in addition to the TLR4/MyD88 pathway, is also an important process (29). It was also previously reported that TRIF knockdown in differentiated neuronal cells decreases the expression of Lox-1 (30). TRIF-dependent pathways may be associated with TLR2 signaling, as it is with TLR-4 signaling. However, the role of the TRIF-dependent pathway in TLR2 signaling has received less investigation.

The results of the present study, which investigated macrophages from mice with TRIF knockdown, provided evidence supporting the connection between TRIF and TLR2 signaling in foam cell formation. The results of the present study indicated that $\mathrm{Pam}_{3} \mathrm{CSK}_{4}$ stimulated macrophage foam cell formation, and induced the expression levels of MCP-1, TF and Lox-1 in the context of an immune response to TLR2 via a TRIF-dependent pathway. In this regard, identification of TRIF as an important regulator of TLR2 signaling in macrophages may represent a possible therapeutic strategy for regulating inflammation and atherosclerosis.

\section{Acknowledgements}

This study was supported by the 2013 Yeungnam University Research Grant (grant no. 213A061034).

\section{References}

1. Lundberg AM, Ketelhuth DF, Johansson ME, Gerdes N, Liu S, Yamamoto M, Akira S and Hansson GK: Toll-like receptor 3 and 4 signalling through the TRIF and TRAM adaptors in haematopoietic cells promotes atherosclerosis. Cardiovasc Res 99: 364-373, 2013.

2. Galkina $E$ and Ley $\mathrm{K}$ : Immune and inflammatory mechanisms of atherosclerosis (*). Annu Rev Immunol 27: 165-197, 2009.

3. Erridge C: The roles of toll-like receptors in atherosclerosis. J Innate Immun 1: 340-349, 2009.

4. Akira S, Uematsu S and Takeuchi O: Pathogen recognition and innate immunity. Cell 124: 783-801, 2006.

5. Verstrepen L, Bekaert T, Chau TL, Tavernier J, Chariot A and Beyaert R: TLR-4, IL-1R and TNF-R signaling to NF-kappaB: Variations on a common theme. Cell Mol Life Sci 65: 2964-2978, 2008.

6. Mogensen TH: Pathogen recognition and inflammatory signaling in innate immune defenses. Clin Microbiol Rev 22: 240-273, 2009.

7. Ahmed S, Maratha A, Butt AQ, Shevlin E and Miggin SM: TRIF-mediated TLR3 and TLR4 signaling is negatively regulated by ADAM15. J Immunol 190: 2217-2228, 2013.

8. Falck-Hansen M, Kassiteridi C and Monaco C: Toll-like receptors in atherosclerosis. Int J Mol Sci 14: 14008-14023, 2013.

9. Michelsen KS, Wong MH, Shah PK, Zhang W, Yano J, Doherty TM, Akira S, Rajavashisth TB and Arditi M: Lack of toll-like receptor 4 or myeloid differentiation factor 88 reduces atherosclerosis and alters plaque phenotype in mice deficient in apolipoprotein E. Proc Natl Acad Sci USA 101: 10679-10684, 2004. 
10. Newton K and Dixit VM: Signaling in innate immunity and inflammation. Cold Spring Harb Perspect Biol 4: a006049, 2012.

11. Curtiss LK and Tobias PS: Emerging role of toll-like receptors in atherosclerosis. J Lipid Res 50 (Suppl): S340-S345, 2009.

12. Mann DL: The emerging role of innate immunity in the heart and vascular system: For whom the cell tolls. Circ Res 108: $1133-1145,2011$.

13. Keyel PA, Tkacheva OA, Larregina AT and Salter RD: Coordinate stimulation of macrophages by microparticles and TLR ligands induces foam cell formation. J Immunol 189: 4621-4629, 2012.

14. Park DW, Lyu JH, Kim JS, Chin H, Bae YS and Baek SH: Role of JAK2-STAT3 in TLR2-mediated tissue factor expression. J Cell Biochem 114: 1315-1321, 2013.

15. Park DW, Baek K, Kim JR, Lee JJ, Ryu SH, Chin BR and Baek SH; Resveratrol inhibits foam cell formation via NADPH oxidase 1-mediated reactive oxygen species and monocyte chemotactic protein-1. Exp Mol Med 41: 171-179, 2009.

16. Lee JG, Lim EJ, Park DW, Lee SH, Kim JR and Baek SH: A combination of Lox-1 and Nox1 regulates TLR9-mediated foam cell formation. Cell Signal 20: 2266-2275, 2008.

17. Mitchell D, Yong M, Schroder W, Black M, Tirrell M and Olive C: Dual stimulation of MyD88-dependent toll-like receptors induces synergistically enhanced production of inflammatory cytokines in murine bone marrow-derived dendritic cells. J Infect Dis 202: 318-329, 2010

18. Dauphinee SM and Karsan A: Lipopolysaccharide signaling in endothelial cells. Lab Invest 86: 9-22, 2006.

19. Oeckinghaus A, Hayden MS and Ghosh S: Crosstalk in NF-кB signaling pathways. Nat Immunol 12: 695-708, 2011.

20. Honda $\mathrm{K}$ and Taniguchi T: IRFs: Master regulators of signalling by toll-like receptors and cytosolic pattern-recognition receptors. Nat Rev Immunol 6: 644-658, 2006.

21. Randall RE and Goodbourn S: Interferons and viruses: An interplay between induction, signalling, antiviral responses and virus countermeasures. J Gen Virol 89: 1-47, 2008.
22. Guijarro-Muñoz I, Compte M, Álvarez-Cienfuegos A, Álvarez-Vallina L and Sanz L: Lipopolysaccharide activates toll-like receptor 4 (TLR4)-mediated NF- $\mathrm{BB}$ signaling pathway and proinflammatory response in human pericytes. J Biol Chem 289: 2457-2468, 2014.

23. Weighardt H, Jusek G, Mages J, Lang R, Hoebe K, Beutler B and Holzmann B: Identification of a TLR4- and TRIF-dependent activation program of dendritic cells. Eur J Immunol 34: 558-564, 2004.

24. Deshmane SL, Kremlev S, Amini S and Sawaya BE: Monocyte chemoattractant protein-1 (MCP-1): An overview. J Interferon Cytokine Res 29: 313-326, 2009.

25. Kundu S, Roome T, Bhattacharjee A, Carnevale KA, Yakubenko VP, Zhang R, Hwang SH, Hammock BD and Cathcart MK: Metabolic products of soluble epoxide hydrolase are essential for monocyte chemotaxis to MCP-1 in vitro and in vivo. J Lipid Res 54: 436-447, 2013.

26. Steffel J, Luscher TF and Tanner FC: Tissue factor in cardiovascular diseases: Molecular mechanisms and clinical implications. Circulation 113: 722-731, 2006.

27. Reiss $\mathrm{AB}$ and Cronstein BN: Regulation of foam cells by adenosine. Arterioscler Thromb Vasc Biol 32: 879-886, 2012.

28. Murphy JE, Vohra RS, Dunn S, Holloway ZG, Monaco AP, Homer-Vanniasinkam S, Walker JH and Ponnambalam S: Oxidised LDL internalisation by the LOX-1 scavenger receptor is dependent on a novel cytoplasmic motif and is regulated by dynamin-2. J Cell Sci 121: 2136-2147, 2008.

29. Xie $H$, Zhou $H$, Wang $H$, Chen $D$, Xia L, Wang $T$ and Yan J: Anti- $\beta(2)$ GPI/ $\beta(2)$ GPI induced TF and TNF- $\alpha$ expression in monocytes involving both TLR4/MyD88 and TLR4/TRIF signaling pathways. Mol Immunol 53: 246-254, 2013.

30. Ding Z, Liu S, Wang X, Khaidakov M, Dai Y, Deng X, Fan Y, Xiang D and Mehta JL: Lectin-like ox-LDL receptor-1 (LOX-1)-toll-like receptor 4 (TLR4) interaction and autophagy in CATH.a differentiated cells exposed to angiotensin II. Mol Neurobiol 51: 623-632, 2015. 